\title{
High-Energy Gamma-ray Observations Using the CALorimetric Electron Telescope
}

\author{
Nicholas Cannady for the CALET Collaboration* \\ Department of Physics \& Astronomy, Louisiana State University, Baton Rouge, LA, United States \\ E-mail: ncannalelsu.edu
}

\begin{abstract}
We present the analysis methodology and preliminary results of the gamma-ray event analysis using the CALorimetric Electron Telescope (CALET). CALET, a 30 radiation length deep calorimeter, was deployed aboard the International Space Station (ISS) in August 2015. In this work we demonstrate the sensitivity of CALET to gamma-rays, the efficiency of separation between gamma-ray and charged particle events, and CALET's exposure on the sky after the first 17 months of observation. Consistency of the flux of the bright sources Geminga and the Crab with established Fermi-LAT results is discussed and initial results of a search for high-energy counterparts to CALET Gamma-ray Burst Monitor gamma-ray bursts are presented.
\end{abstract}

35th International Cosmic Ray Conference -ICRC2017-

10-20 July, 2017

Bexco, Busan, Korea

\footnotetext{
*Speaker.
} 


\section{Introduction}

The CALET instrument $[1,2]$ docked with the ISS in August 2015 and has been in scientific data-taking operations since October 2015. CALET consists of two main instruments: the calorimeter (CAL) and the CALET Gamma-ray Burst Monitor (CGBM). CALET-CAL is a $30 \mathrm{X}_{0}$ (radiation length) deep, imaging calorimeter (Fig. 1) capable of measuring the cosmic ray electron spectrum from $1 \mathrm{GeV}$ to $20 \mathrm{TeV}$, gamma-rays from $1 \mathrm{GeV}$ to $10 \mathrm{TeV}$, and protons and nuclei up to $1 \mathrm{PeV}$. CGBM [3] is a combined $\mathrm{LaBr}_{3}-\mathrm{BGO}$ telescope sensitive from $7 \mathrm{keV}$ to $20 \mathrm{MeV}$. This paper briefly describes the performance of the CALET calorimeter on-orbit, the methodology for gamma-ray event identification, and presents the initial results of 17 months of observations.

\section{CALET-CAL}

CAL is capable of high-energy measurements of gamma-rays from steady and transient sources with fine angular and energy resolution. It has three subdetectors, as shown in Fig. 1: the CHarge Detector (CHD), the IMaging Calorimeter (IMC), and the Total AbSorption Calorimeter (TASC). CHD comprises two crossed layers of 14 plastic scintillator paddles (32 mm x $450 \mathrm{~mm}$ x $10 \mathrm{~mm}$ ), allowing for precise measurement of the incident particle absolute charge with a resolution of 0.15 - 0.3 e $[1,2]$. The IMC contains 8 pairs of layers, each with 2 x 448 plastic scintillator fibers $(1$ $\mathrm{mm} \times 1 \mathrm{~mm} \times 448 \mathrm{~mm}$ ). Interspersed in the IMC layers are 7 sheets of tungsten (upper 5: $448 \mathrm{~mm}$ x $448 \mathrm{~mm}$ x $0.7 \mathrm{~mm}$, lower 2: $448 \mathrm{~mm}$ x $448 \mathrm{~mm}$ x $3.5 \mathrm{~mm}$ ) for stimulating shower development. The IMC is capable of imaging the electromagnetic shower development and allows for tracking with angular resolution finer than $0.5^{\circ}$ for gamma-rays with energies $3 \mathrm{GeV}$ to $10 \mathrm{TeV}$ [4]. Finally, the TASC is composed of $192 \mathrm{PbWO}_{4} \operatorname{logs}(19 \mathrm{~mm} \times 326 \mathrm{~mm} \times 20 \mathrm{~mm}$ ) that enable measurement of the energy of electron or photon primaries with energy resolution 2\% [5].

CAL operates on-orbit in two trigger modes, the High-Energy trigger (HE) and the Lowenergy Gamma trigger (LEG). The HE trigger is operated with a nearly 100\% duty fraction, being disabled only for passage through the SAA, and enables measurement of electrons and gamma-ray events with energies above $\sim 10 \mathrm{GeV}$. The LEG trigger is sensitive to primary energies down to $\sim 1$ $\mathrm{GeV}$, but is only active at low geomagnetic latitudes (where the rigidity cutoff is used to reduce contamination from charged particles) and following gamma-ray bursts (GRB) seen by CGBM.

CALET was calibrated on the ground using penetrating muons, gamma-ray sources, and UV laser light. On orbit, calibration of the instrument is performed using the minimum-ionizing particle (MIP) signal from penetrating He ions along with environmental measurements (temperature, solar beta angle, etc.) and established detector characteristics. The calibration process is described in detail in [5], achieving relative error $<2 \%$ for $\mathrm{E}>10 \mathrm{GeV}$ and $<10 \%$ for $\mathrm{E}>1 \mathrm{GeV}$.

\section{Gamma-ray Event Selection}

The requirements for preselection and identification of photon events and the corresponding efficiencies as a function of energy, are derived using the EPICS/Cosmos simulation packages ${ }^{1}$.

\footnotetext{
${ }^{1}$ The EPICS and Cosmos packages are available from http://cosmos.n.kanagawa-u.ac.jp/
} 


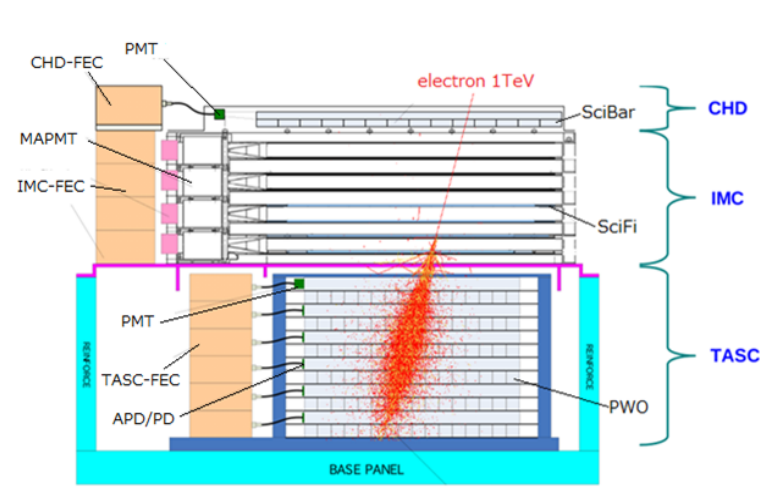

Figure 1: A schematic of the physical layout of the CALET-CAL, including a trace of a simulated $1 \mathrm{TeV}$ electron.

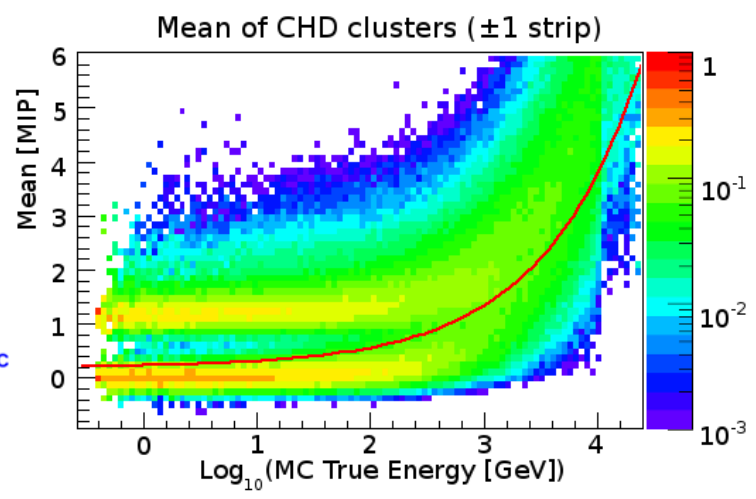

Figure 2: Simulated distribution of CHD cluster means for electrons and gamma-rays passing preselection conditions. The red line denotes the cho$\operatorname{sen} \mathrm{Z}=0$ cut as a function of energy.

The on-board LEG and HE triggers require thresholds on the IMC 7, IMC 8, and TASC X1 layers $[1,2]$. In the IMC, the photomultiplier tube dynode sums are used (IMC $7 \mathrm{X}$ and $8 \mathrm{X}$ are paired, as are IMC 7Y and 8Y). For the LEG trigger, the thresholds are set to $\sim 2.5$ MIP in each IMC dynode and $\sim 3$ MIP in the TASC X1 dynode sum. The on-board HE trigger requires $~ 25$ MIP and $\sim 75$ MIP in the IMC dynodes and the TASC X1 dynodes, respectively.

A series of offline filters are applied to the data in order to isolate gamma-ray candidates and require agreement between the simulated and flight datasets. The offline trigger mimics the online trigger with increased thresholds. To guarantee clean charge measurement, events are required to be reconstructed with the EM Track [6] or CCTrack tracking algorithms and to satisfy reliability conditions on the number of layers used and the reduced $\chi^{2}$ statistic of the fit. The acceptance conditions for the trajectory require that the primary particle enter the instrument through the top of the CHD for charge identification and pass through the bottom of the TASC to allow for accurate energy reconstruction. Contamination from proton events and events entering the side of the CAL is reduced by requiring the fraction of energy deposited within $9 \mathrm{~mm}$ of the projected intersection of the track with the layer plane for the lower IMC layers (8X, 8Y) near the track be at least $50 \%$.

With these preselection conditions in place, the isolation of $\mathrm{Z}=0$ events is straightforward. An upper threshold is placed at 0.1 MIP on the mean of the signals in the hit clusters (hit fiber \pm 2 ) in the $\mathrm{X}$ and $\mathrm{Y}$ layers of IMC1. Similarly, a limit is placed on the mean of the signals in the CHDx and CHDy clusters (hit strip \pm 1 ). Based on the distributions seen in simulated data, the CHD threshold is energy dependent and follows the empirical form $S_{\text {cut }}[\mathrm{MIP}]=0.2+\sqrt{\frac{E}{3 \mathrm{TeV}}}$ (Fig. 2).

Efficiencies are calculated as a function of energy using the simulated dataset from EPICS/Cosmos by applying the offline trigger, tracking requirements, and charge selection as described above. The efficiency for detection rises with increasing energy from $\sim 10 \%$ at $1 \mathrm{GeV}$ (primarily limited by the trigger), reaches a maximum of $40-45 \%$ in the energy range 10 to $100 \mathrm{GeV}$, and falls slowly for energies $>100 \mathrm{GeV}$ due to backscattered particles from interactions deeper in the calorimeter contaminating the charge measurement in the CHD (Fig. 2). 


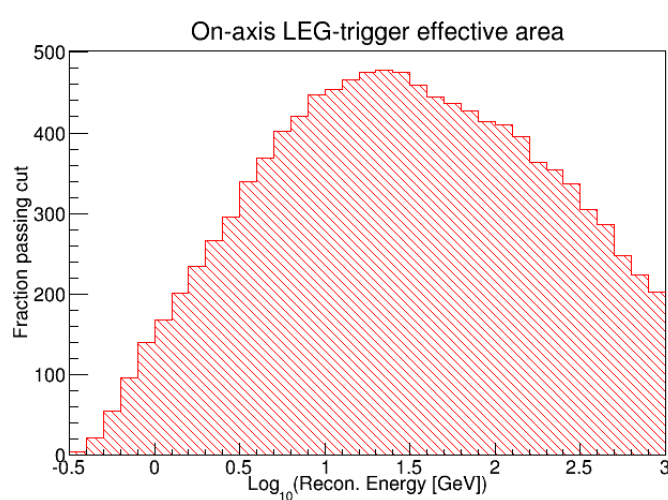

Figure 3: Effective area for the event geometry considered in this work.

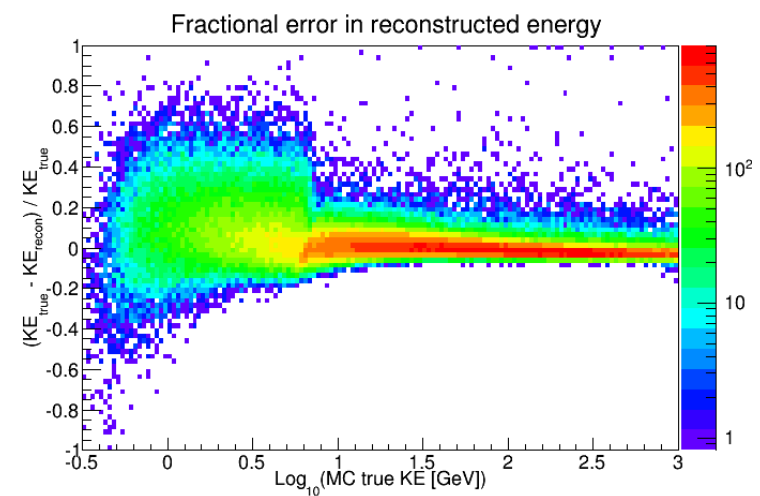

Figure 4: Error in primary energy reconstruction from simulated $\mathrm{e}^{-}$and photons.

\section{Performance as a Gamma-ray Telescope}

The angular resolution of the CAL is determined by the accuracy of the tracking algorithms and can be evaluated using the reconstructed arrival directions of photons from known point sources. Prior to launch, the CAL angular resolution $\left(\sigma_{P S F}\right)$ was calculated via simulation [4] to be approximately $0.3^{\circ}$ for photons with $\mathrm{E}>10 \mathrm{GeV}$ and decreasing below $10 \mathrm{GeV}$. In flight, we evaluate the angular resolution by analyzing the PSF of signals from known point sources, finding a fluxaveraged angular resolution of $\sim 0.4^{\circ}$ from photons associated with the Crab (cf. Sec. 5 below).

The prescription for geometric area as a function of incident direction for two square surfaces follows [7], and is dependent on both the zenith and azimuth angles of the incident photons in the detector frame. The effective area is then determined from simulations based on the acceptance conditions for event trajectories and the efficiency for detection, tracking, and positive photon identification as a function of energy (Fig. 3).

Photon energies are determined by summing the signals from the PWO logs in the TASC. Above $\sim 6 \mathrm{GeV}$, a normalization factor of 1.075 is applied (based on simulations) to calculate the energy of photon candidates with an accuracy better than a few percent. Below this energy, the contribution of pedestal fluctuations in the TASC channels becomes a significant fraction of the signal and a limited region around the reconstructed track is used. The scaling factor for these events is $\sim 25 \%$, and the uncertainty in the reconstructed energy is on the order of $10 \%$. The fractional error in the energy reconstruction is shown in Fig. 4.

\section{Gamma-ray Observations}

Photon candidates are extracted from the flight data based on passing the preselection conditions and the $\mathrm{Z}=0$ requirement detailed above. The arrival direction is calculated using the incidence angles of the reconstructed trajectory and calibrated rotation quaternions derived from the CALET Advanced Stellar Compass (ASC) or, if ASC images are not available, from the ISS telemetry. An exposure map is generated by projecting the geometric area scaled by the live time onto the sky using the HEALPix sky pixelization scheme ${ }^{2}$ for each event where the LEG trigger was active. The

\footnotetext{
2 http://healpix.sourceforge.net
} 


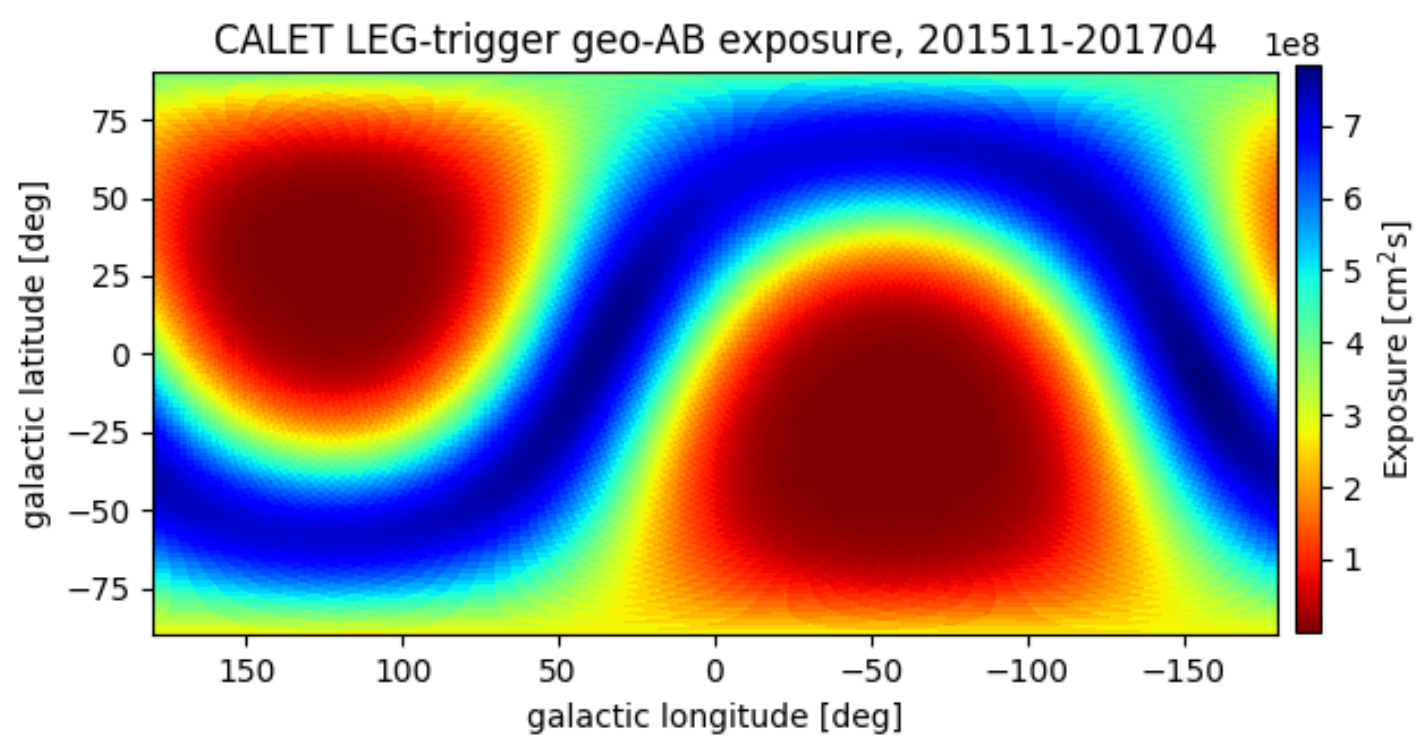

Figure 5: Exposure of the CALET LEG trigger for events with trajectories crossing the top of the CHD and the bottom of the TASC calculated for 2015/11/01 through 2017/04/20.
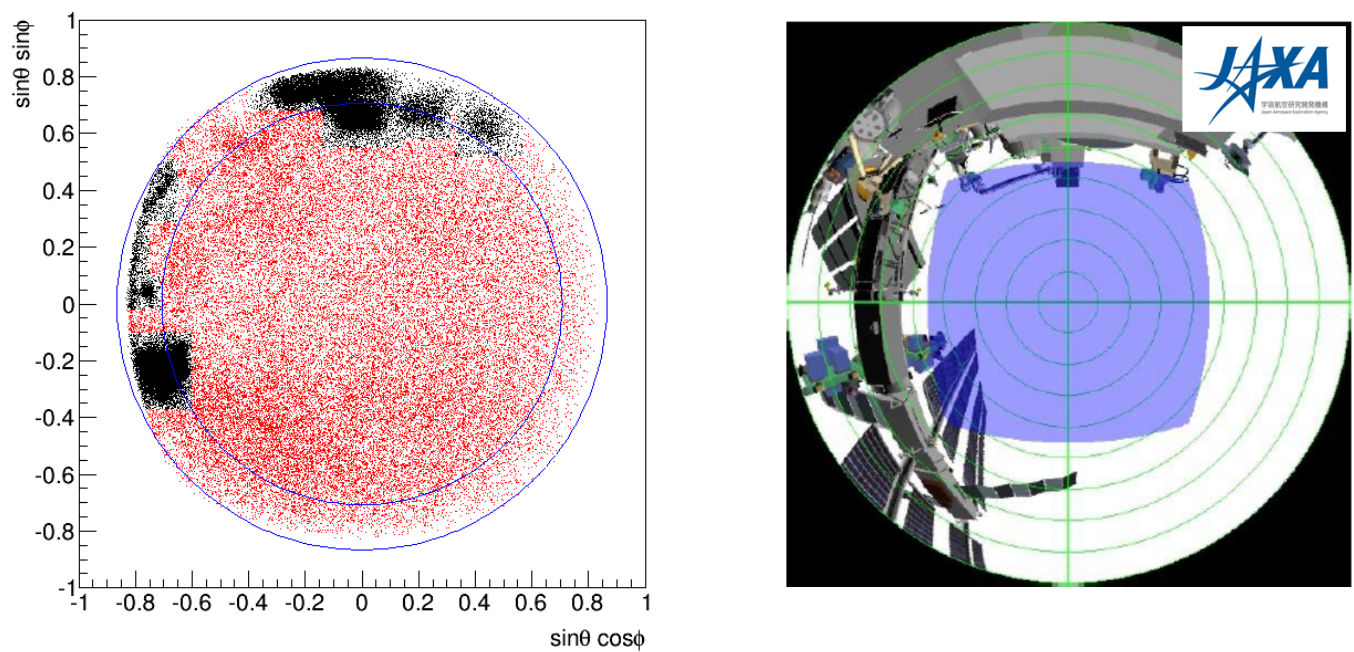

Figure 6: ISS structure obstructing the field of view of the CAL. The left scatter plot shows photon candidates in the CAL detector frame, with those in the masked regions in black. The image on the right shows a snapshot of the ISS structures from the perspective of the CAL.

exposure map for all LEG trigger observations from 2015/11 to 2017/04 is shown in Fig. 5.

An important consideration for background for gamma-ray observations on-board the ISS are structures present in the field of view. As seen in Fig. 6, solar panels and metal structures are present in the CAL field of view beyond a zenith angle of $\sim 45^{\circ}$. Since fluorescence $\mathrm{x}$-rays and scattered gamma-rays from these structures create a source of background for CAL measurements, these regions are masked for event rejection.

Based on the exposure in Fig. 5 and the region masking in 6, photon candidate positions are 


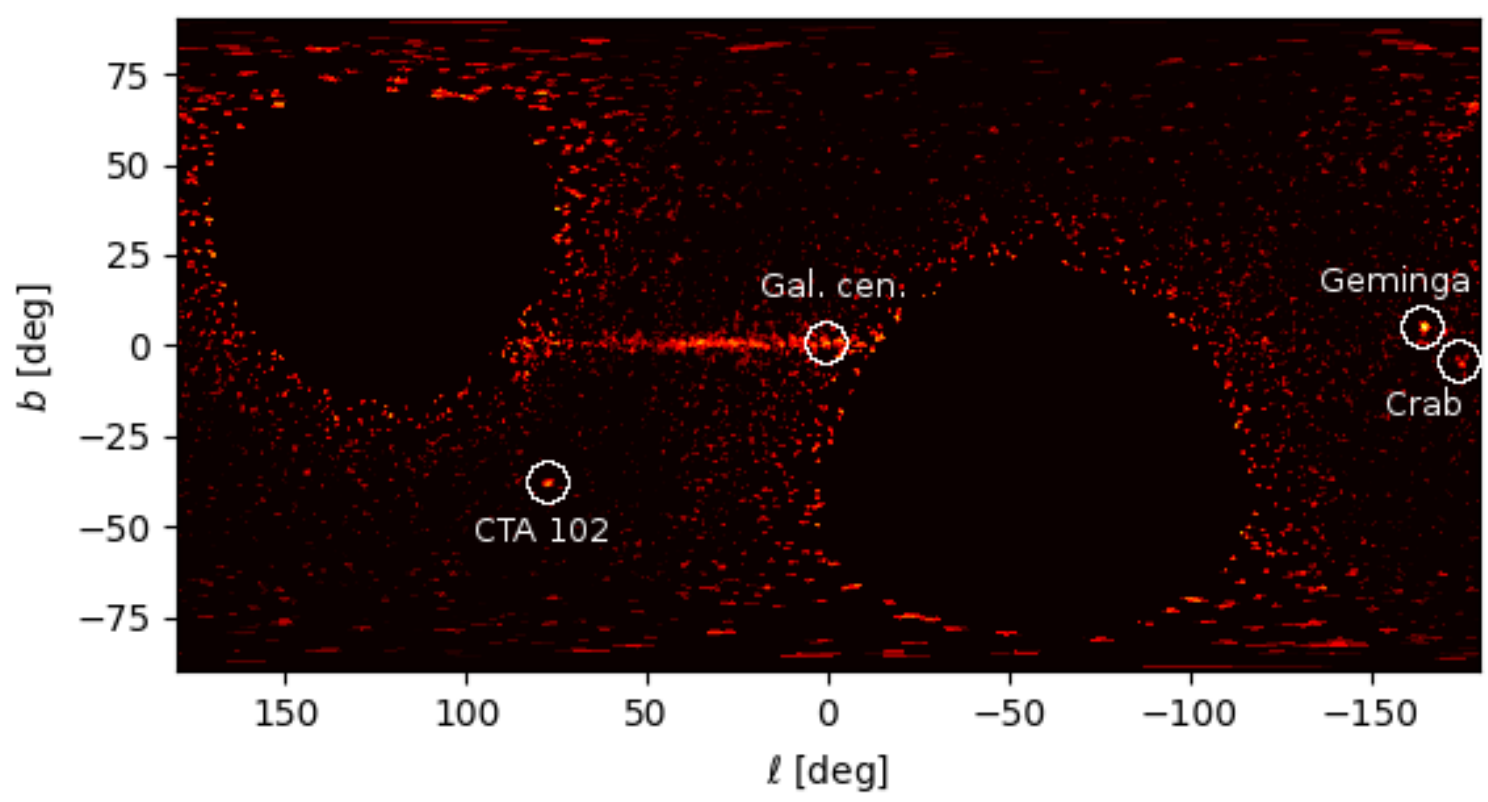

Figure 7: Sky map showing the counts scaled to exposure for the same observation time and acceptance conditions as Fig. 5. White circles indicate source positions for (from left) AGN CTA 102 (c.f. Fig. 11), the galactic center, Geminga, and the Crab.

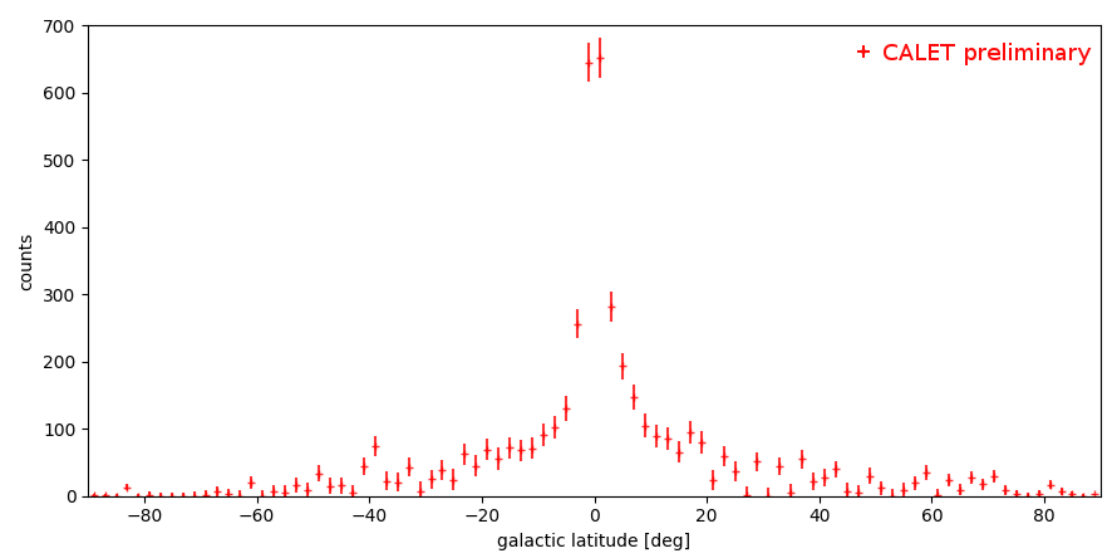

Figure 8: Distribution of photon candidates vs. galactic latitude for $|\ell|<80^{\circ}$. CAL data used spans 17 months, from start of scientific operations through 2017/03.

shown in Fig. 7 for the LEG trigger from 2015/11 to 2017/04.

Fig. 8 shows the galactic latitude distribution of photon candidates seen by CAL over the first 17 months of observation. Charged particle-induced background and the contribution of the isotropic gamma-ray flux have been preliminarily removed as a component that scales directly with exposure. Detailed study of the background contamination and a comparison with the expected signal from the Fermi-LAT galactic diffuse emission model [8] are in progress.

The Crab can be clearly seen in Fig. 7 and can be used to confirm the in-flight angular resolution performance. For a selection of events from the region of the Crab, Fig. 9 shows the angular distance from the known source position as given by SIMBAD [9], with a measured $\sigma_{P S F}=0.4^{\circ}$. 


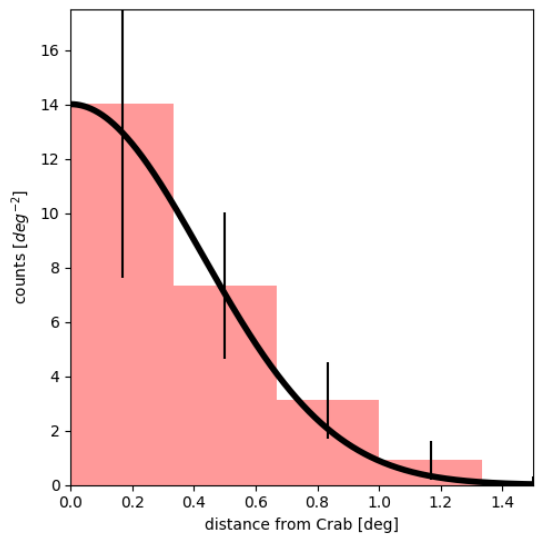

Figure 9: Number of photon candidates vs. distance from the SIMBAD Crab position. The solid line represents a Gaussian fit to the PSF, with $\sigma=0.4^{\circ}$.

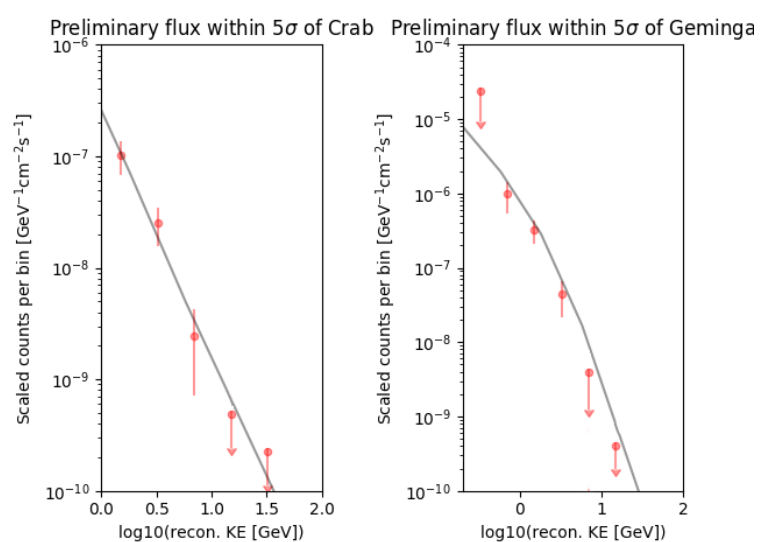

Figure 10: Flux from Crab and Geminga in 17 months of CAL flight data with the results from Fermi-LAT 3FGL. Black lines are from the LAT, and red points are from CAL. Errorbars are statistical only.

Fig. 10 shows the fluxes measured for the Crab and Geminga. Photons associated with the sources are chosen to lie within a distance of $5 \sigma_{P S F}$ of the known source positions. The differential flux is calculated by summing the photon candidates weighted by the CAL detection efficiency and scaling to the exposure on the sky for the source position and energy bin width. For the Crab, the exposure is calculated to be $\sim 4 \times 10^{8} \mathrm{~cm}^{2}$ s, with 46 photon candidates associated with the source position. For Geminga, the exposure is $\sim 5 \times 10^{8} \mathrm{~cm}^{2} \mathrm{~s}$, with 119 associated photon candidates. The solid curves in Fig. 10 show the Fermi-LAT 3FGL spectra for comparison [10].

Transient activity from the bright radio blazar CTA 102 [11] was also observed by CAL. Fig. 11 shows the flux measured in the CAL as the AGN begins to rise in 2016/11, reaches a peak in $2016 / 12$, and begins to fade in 2017/01. Smaller flares are seen in 2017/02 and 2017/04. The LAT light curve $^{3}$ is shown in Fig. 12 for comparison (red lines separate months of interest). Analysis is ongoing to increase the field of view of CAL for CTA 102 and similar transients.

\section{Search for Transient Counterparts}

CAL is able to search for and place upper limits on the fluxes $>1 \mathrm{GeV}$ of high-energy counterparts to transient events. The results of CGBM and CAL searches for electromagnetic counterparts to LIGO- Virgo gravitational wave triggers can be found in [12] and in these proceedings [13]. In addition, the gamma-ray observations from the CAL have been searched for potential high-energy signals correlated spatially and/or temporally with GRB triggers in CGBM and other instruments. The background for a given signal is estimated by sampling the orbit in the same sky location as the GRB localization (if available) when there is no trigger. At present, an analysis of times within $\pm 60 \mathrm{sec}$. of CGBM GRB triggers shows no evidence of high-energy CAL counterparts.

\footnotetext{
${ }^{3}$ https://fermi.gsfc.nasa.gov/ssc/data/access/lat/msl_lc/source/CTA_102
} 


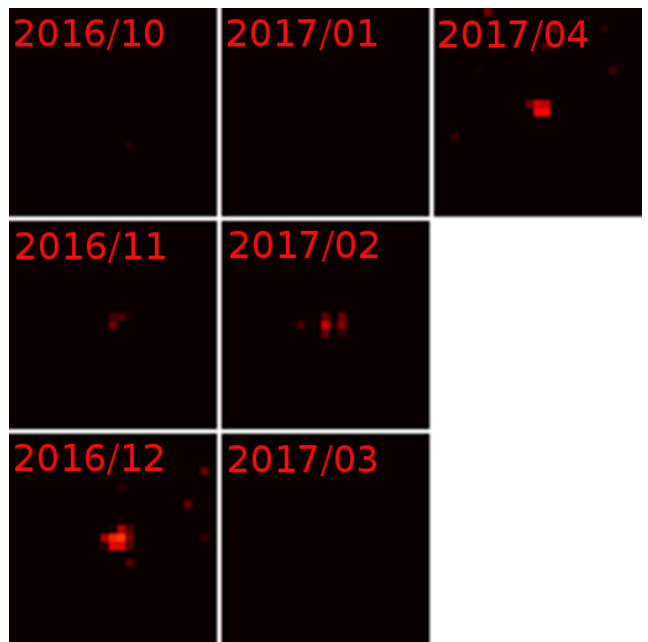

Figure 11: CAL observations of CTA 102 as it exhibited flaring activity in late 2016 to early 2017.

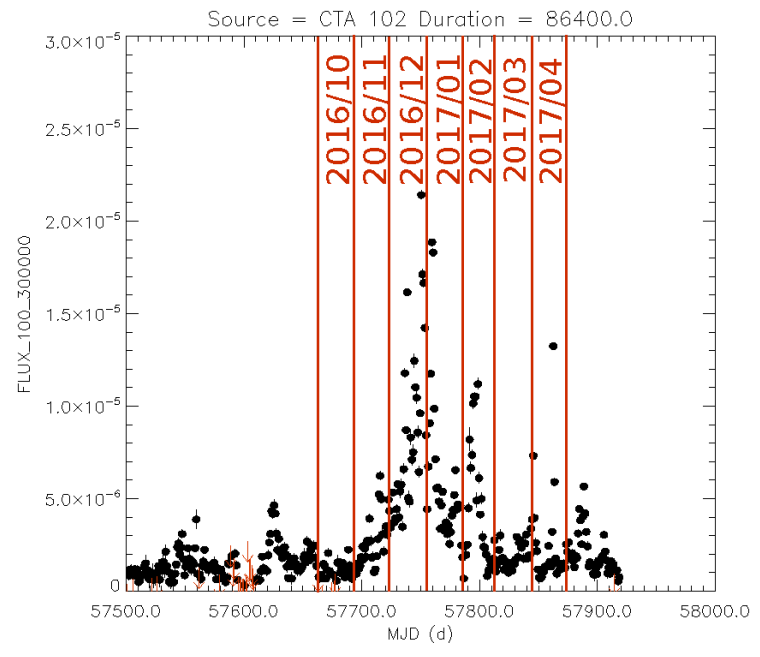

Figure 12: LAT light curve for CTA 102 showing the increased activity in late 2016 to early 2017.

\section{Acknowledgements}

This work was supported in Japan by JAXA, in Italy by ASI, and in the U.S. by NASA. The author appreciates support provided by the Louisiana Board of Regents. The CERN ROOT ${ }^{4}$ framework (R. Brun and F. Rademakers, NIM. in Phys. Res. A, 389 (1997)) and the HEALPix package (K.M. Gorski et al., ApJ, 622 (2005)) were used in deriving some results in this work.

\section{References}

[1] Torii, S. for the CALET Collaboration, in Proc. $33^{\text {rd }}$ ICRC, Rio de Janeiro (2013).

[2] Torii, S. for the CALET Collaboration, these proceedings.

[3] Yamaoka, K. for the CALET Collaboration, these proceedings \#614.

[4] Mori, M. for the CALET Collaboration, in Proc. $33^{\text {rd }}$ ICRC, Rio de Janeiro (2013).

[5] Asaoka, Y. et al., Astroparticle Physics, 91, 1 (2017).

[6] Akaike, Y. for the CALET Collaboration, in Proc. $33^{\text {rd }}$ ICRC, Rio de Janeiro (2013).

[7] Sullivan, J.D., Nucl. Instrum. Meth., 95, 5 (1971).

[8] Acero, F. et al., Ap.J. Supplement Series, 223:26 (2016).

[9] Wenger et al., A\&AS, 143:9, (2000).

[10] Acero, F. et al., Ap.J. Supplement Series, 218:23 (2015).

[11] Ciprini, S. et al., ATel No. 9869 (2016).

[12] Adriani, O. et al., Ap.J. Letters, 829, L20 (2016).

[13] Mori, M. for the CALET Collaboration, these proceedings \#637.

\footnotetext{
${ }^{4}$ http://root.cern.ch
} 